\title{
Glassy low-energy spin fluctuations and anisotropy gap in La1.88Sr0.12CuO4
}

Rømer, A. T.; Chang, J.; Christensen, Niels Bech; Andersen, B. M.; Lefmann, K.; Mähler, L.; Gavilano, J.; Gilardi, R.; Niedermayer, Ch; Rønnow, H. M.

Total number of authors:

16

Published in:

Physical Review B Condensed Matter

Link to article, DOI:

10.1103/PhysRevB.87.144513

Publication date:

2013

Document Version

Publisher's PDF, also known as Version of record

Link back to DTU Orbit

Citation $(A P A)$ :

Rømer, A. T., Chang, J., Christensen, N. B., Andersen, B. M., Lefmann, K., Mähler, L., Gavilano, J., Gilardi, R., Niedermayer, C., Rønnow, H. M., Schneidewind, A., Link, P., Oda, M., Ido, M., Momono, N., \& Mesot, J. (2013). Glassy low-energy spin fluctuations and anisotropy gap in $\mathrm{La}_{1}{ }_{18} \mathrm{Sr}_{1} \mathrm{C}_{12} \mathrm{CuO}_{4}$. Physical Review $\mathrm{B}$ Condensed

\section{General rights}

Copyright and moral rights for the publications made accessible in the public portal are retained by the authors and/or other copyright owners and it is a condition of accessing publications that users recognise and abide by the legal requirements associated with these rights.

- Users may download and print one copy of any publication from the public portal for the purpose of private study or research.

- You may not further distribute the material or use it for any profit-making activity or commercial gain

- You may freely distribute the URL identifying the publication in the public portal 


\title{
Glassy low-energy spin fluctuations and anisotropy gap in $\mathrm{La}_{1.88} \mathrm{Sr}_{0.12} \mathrm{CuO}_{4}$
}

\author{
A. T. Rømer, ${ }^{1,2}$ J. Chang,,${ }^{3,45}$ N. B. Christensen, ${ }^{6}$ B. M. Andersen, ${ }^{1}$ K. Lefmann, ${ }^{1}$ L. Mähler, ${ }^{3}$ J. Gavilano, ${ }^{3}$ R. Gilardi, ${ }^{3}$ \\ Ch. Niedermayer, ${ }^{3}$ H. M. Rønnow, ${ }^{2}$ A. Schneidewind, ${ }^{7}$ P. Link, ${ }^{8}$ M. Oda, ${ }^{9}$ M. Ido, ${ }^{9}$ N. Momono, ${ }^{10}$ and J. Mesot ${ }^{3,4,5}$ \\ ${ }^{1}$ Niels Bohr Institute, University of Copenhagen, DK-2100 Copenhagen, Denmark \\ ${ }^{2}$ Laboratory for Quantum Magnetism, École Polytechnique Fédérale de Lausanne (EPFL), 1015 Lausanne, Switzerland \\ ${ }^{3}$ Laboratory of Neutron Scattering, Paul Scherrer Institute, 5232-Villigen, Switzerland \\ ${ }^{4}$ Laboratory for Solid State Physics, ETH Zürich, CH-8093 Zürich, Switzerland \\ ${ }^{5}$ Laboratory for Synchrotron and Neutron Spectroscopy, École Polytechnique Fédérale de Lausanne (EPFL), 1015 Lausanne, Switzerland \\ ${ }^{6}$ Department of Physics, Technical University of Denmark, DK-2800 Kgs. Lyngby, Denmark \\ ${ }^{7}$ Institut für Festkörperphysik (IFP), Technische Universität Dresden, D-01062 Dresden, Germany \\ ${ }^{8}$ Forschungsneutronenquelle Heinz Maier-Leibnitz, (FRM II), Technische Universität München, 85747 Garching, Germany \\ ${ }^{9}$ Department of Physics, Hokkaido University, Sapporo 060-0810, Japan \\ ${ }^{10}$ Department of Applied Sciences, Muroran Institute of Technology, Muroran 050-8585, Japan \\ (Received 22 February 2013; revised manuscript received 4 April 2013; published 22 April 2013)
}

\begin{abstract}
We present high-resolution triple-axis neutron scattering studies of the high-temperature superconductor $\mathrm{La}_{1.88} \mathrm{Sr}_{0.12} \mathrm{CuO}_{4}\left(T_{c}=27 \mathrm{~K}\right)$. The temperature dependence of the low-energy incommensurate magnetic fluctuations reveals distinctly glassy features. The glassiness is confirmed by the difference between the ordering temperature $T_{N} \simeq T_{c}$ inferred from elastic neutron scattering and the freezing temperature $T_{f} \simeq 11 \mathrm{~K}$ obtained from muon spin rotation studies. The magnetic field independence of the observed excitation spectrum as well as the observation of a partial suppression of magnetic spectral weight below $0.75 \mathrm{meV}$ for temperatures smaller than $T_{f}$, indicate that the stripe frozen state is capable of supporting a spin anisotropy gap, of a magnitude similar to that observed in the spin and charge stripe-ordered ground state of $\mathrm{La}_{1.875} \mathrm{Ba}_{0.125} \mathrm{CuO}_{4}$. The difference between $T_{N}$ and $T_{f}$ implies that the significant enhancement in a magnetic field of nominally elastic incommensurate scattering is caused by strictly inelastic scattering —at least in the temperature range between $T_{f}$ and $T_{c}$ —which is not resolved in the present experiment. Combining the results obtained from our study of $\mathrm{La}_{1.88} \mathrm{Sr}_{0.12} \mathrm{CuO}_{4}$ with a critical reappraisal of published neutron scattering work on samples with chemical composition close to $p=0.12$, where local probes indicate a sharp maximum in $T_{f}(p)$, we arrive at the view that the low-energy fluctuations are strongly dependent on composition in this regime, with anisotropy gaps dominating only sufficiently close to $p=0.12$ and superconducting spin gaps dominating elsewhere.
\end{abstract}

DOI: 10.1103/PhysRevB.87.144513

PACS number(s): 74.72.-h, 75.25.-j, 75.40.Gb, 78.70.Nx

\section{INTRODUCTION}

In the presence of quenched disorder, competing order parameters in strongly correlated electron systems are known to result in interesting physical phenomena such as phase separation, glassiness, and dramatic responses to applied stimuli. ${ }^{1}$ Doped transition metal oxides are perhaps the most studied examples of these general themes. Notably, high-temperature superconducting cuprates have attracted enormous interest since their discovery. ${ }^{2}$ Undoped cuprates are charge transfer insulators that upon charge-carrier doping of the $\mathrm{CuO}_{2}$ layers become superconducting. For carrier concentrations lower than optimal for superconductivity, i.e., in the underdoped regime, several competing or coexisting order parameters have been identified, such as circulating orbital currents, ${ }^{3-5}$ incommensurate spin and charge stripe ordering, ${ }^{6,8}$ and recently, charge density wave order. 9,10 Stripe order in cuprates was originally discovered when the hole-doping level $p$ was tuned to $p=x=1 / 8$ in $\mathrm{La}_{1.6-x} \mathrm{Nd}_{0.4} \mathrm{Sr}_{x} \mathrm{CuO}_{4}$ (Nd-LSCO). ${ }^{8}$ Subsequently, stripe order has been observed in $\mathrm{La}_{2-x} \mathrm{Ba}_{x} \mathrm{CuO}_{4}$ $(\mathrm{LBCO})^{6,7}$ and $\mathrm{La}_{1.8-x} \mathrm{Eu}_{0.2} \mathrm{Sr}_{x} \mathrm{CuO}_{4}$ (Eu-LSCO), ${ }^{11,12}$ at $p=$ $1 / 8$. In all three cases, competition between incommensurate spin-charge order and superconductivity causes a dramatic drop of $T_{c}$, which reaches very low values at $x=1 / 8$, where the stripe-ordering tendencies are most pronounced. Even in the archetypal superconductor $\mathrm{YBa}_{2} \mathrm{Cu}_{3} \mathrm{O}_{y}$ (YBCO) that has an optimal $T_{c}=90 \mathrm{~K}$, a small suppression of the superconducting transition temperature has been found near the $1 / 8$ doping. ${ }^{13}$ In the case of $\mathrm{YBCO}$, the exact nature of the competing order parameter is still being explored, with the most recent evidence from NMR, ${ }^{14}$ transport, ${ }^{15}$ and $\mathrm{X}$-ray scattering techniques ${ }^{9,10}$ pointing to charge-density wave order. $\mathrm{La}_{2-x} \mathrm{Sr}_{x} \mathrm{CuO}_{4}$ (LSCO) falls in between YBCO and the stripe compounds Nd-LSCO and LBCO. Like YBCO, it displays only a small suppression of $T_{c}$ in the vicinity of $p=$ $x=1 / 8,{ }^{16}$ but in this regime, incommensurate magnetism similar to that found for Nd-LSCO and LBCO coexists with superconductivity in the ground state. ${ }^{17}$ To date, no evidence for incommensurate bulk charge order in LSCO has emerged. ${ }^{18}$ This difference is believed to be an effect of a favorable potential for charge-stripe pinning in the specific low-temperature tetragonal (LTT) structure of Nd-LSCO and LBCO. Within this picture, the low-temperature orthorhombic (LTO) structure of LSCO is not suitable for charge stripe order, but does allow incommensurate magnetism near $x=1 / 8$. $^{17,19}$ The onset temperature of the incommensurate magnetism in LSCO depends on the experimental technique used to probe it. This implies that the electronic spins are gradually freezing rather than undergoing a regular thermodynamic phase transition. For LSCO the freezing temperature $T_{f}$, derived from local probes such as NMR, NQR and muon spin rotation $(\mu \mathrm{SR})$ has a narrow peak centered around $x_{\max } \sim 0.12 .^{20}$

It appears reasonable to conjecture that the details of the magnetic excitation spectrum may be highly sensitive to 
doping near $x_{\max }$. The available experimental evidence is, however, limited and a consistent interpretation is lacking. A recent doping-dependent study of the low-energy dynamics in LSCO by M. Kofu et al. ${ }^{21}$ reported a correlation between the presence of incommensurate elastic magnetic scattering and gapless spin excitations near $x_{\max }$. These data were interpreted in terms of two components: a spin-gapped response similar to what is observed at optimal doping, ${ }^{22}$ i.e., a gap caused by superconductivity, and a second component related to spin stripe-ordered or stripe-frozen domains. In contrast to this view, J. Chang et al. ${ }^{23}$ proposed that for $x=0.105<x_{\max }$ magnetic order renormalizes the value of the superconductivity-related spin gap. The two-component view is also in contrast to what is observed at $x=0.145>x_{\max }$ where the spin gap is found to close at the quantum critical point for the incommensurate spin order. ${ }^{24,25}$

Here, we present results of experiments designed to improve our understanding of the low-energy dynamic magnetic fluctuations in LSCO and move towards a consistent description of its doping-dependence near $x_{\max }$. We have studied the temperature and magnetic field-dependence of the spin-dynamics of LSCO at $x=0.12 \simeq x_{\max }$. In contrast to the clear effects of magnetic field on low-energy excitations reported both for the underdoped regime for $x<x_{\max }{ }^{23}$ and for the optimally doped regime for $x>x_{\max },{ }^{24,26-28}$ we observe no field effect for $x \simeq x_{\max }$. The temperature dependence of the spin fluctuations reveals glassy dynamics and, at the lowest-energy transfers, a partial suppression of magnetic spectral weight below $0.75 \mathrm{meV}$. The latter observation in combination with the absence of a magnetic field effect on the low-energy excitations, suggests that LSCO near $x_{\max }$ can support a spin anisotropy gap despite the glassy nature of the ordering and despite superconductivity. We discuss the implications of our results and their relation to previously published work on $\mathrm{La}_{2-x} \mathrm{Sr}_{x} \mathrm{CuO}_{4}, \mathrm{La}_{2-x} \mathrm{Ba}_{x} \mathrm{CuO}_{4}$, and spincharge ordered nickelates $\mathrm{La}_{2-x} \mathrm{Sr}_{x} \mathrm{NiO}_{4}$ in Sec. IV. Here, we arrive at a validation of the conjecture that the low-energy excitations are strongly dependent on hole-content near the maximum in $T_{f}$. First, however, we describe the experimental methods in Sec. II and present our experimental data in Sec. III.

\section{EXPERIMENTAL METHOD}

The $\mathrm{La}_{1.88} \mathrm{Sr}_{0.12} \mathrm{CuO}_{4}\left(T_{\mathrm{c}} \approx 27 \mathrm{~K}\right)$ sample studied consisted of two single crystals that were cut from the same rod grown by the traveling solvent floating method. ${ }^{29}$ In earlier work, ${ }^{19}$ the Sr content $x=0.120 \pm 0.005$ (and hence the hole concentration $p=x$ ) was determined from the structural transition temperature separating the high-temperature tetragonal (HTT) from the low-temperature orthorhombic (LTO) phase. Muon spin rotation studies on one of the two single crystals revealed electronic moments that are static on the muon time scale below a freezing temperature $T_{f} \approx 11 \mathrm{~K} .{ }^{19,30}$ High-resolution inelastic neutron scattering experiments were carried out on the PANDA cold neutron triple axis spectrometer at the FRM-II research neutron source in Munich, Germany. In a first experiment, the two crystal rods were co-aligned to within less than one degree and the sample was inserted in a $15-\mathrm{T}$ vertical field cryomagnet and the instrument configured with vertically focusing pyrolytic graphite (PG) monochromator and collimation sequence open-60'-open-open from source to detector. In a second, zero-field, experiment only one crystal rod was used in a setup with a double-focusing monochromator and no collimation. In both experiments, a cooled Be-filter was placed in front of the double-focusing PG analyzer to minimize contamination from higher-order neutrons. The sample was oriented with the crystallographic $c$ axis vertical, allowing access to wave vectors of the form $Q=(H, K, 0)$. In labeling reciprocal space, it is convenient to use notation corresponding to the high-temperature tetragonal crystal structure ( $a \simeq b=$ $3.78 \AA, c \simeq 13.18 \AA$ ). In this notation, the propagation vector of the undoped antiferromagnetic parent compound $\mathrm{La}_{2} \mathrm{CuO}_{4}{ }^{31}$ is $(1 / 2,1 / 2,0)$ while stripe magnetic ordering ${ }^{8}$ is manifested in a quartet of peaks, incommensurate with the lattice at $Q_{\mathrm{IC}}=(1 / 2 \pm \delta, 1 / 2, L)$ and $(1 / 2,1 / 2 \pm \delta, L)$ [see the inset in Fig. 1(a)]. For our sample, $\delta=0.125(3)$ as reported earlier. ${ }^{19}$ The intensity recorded by the neutron detector is the convolution of the instrumental resolution function with the spin-spin correlation function $S(\mathbf{Q}, \omega, T)$, which in turn is related to the imaginary part of the generalized magnetic susceptibility $\chi^{\prime \prime}(\mathbf{Q}, \omega, T)$ via the fluctuation dissipation

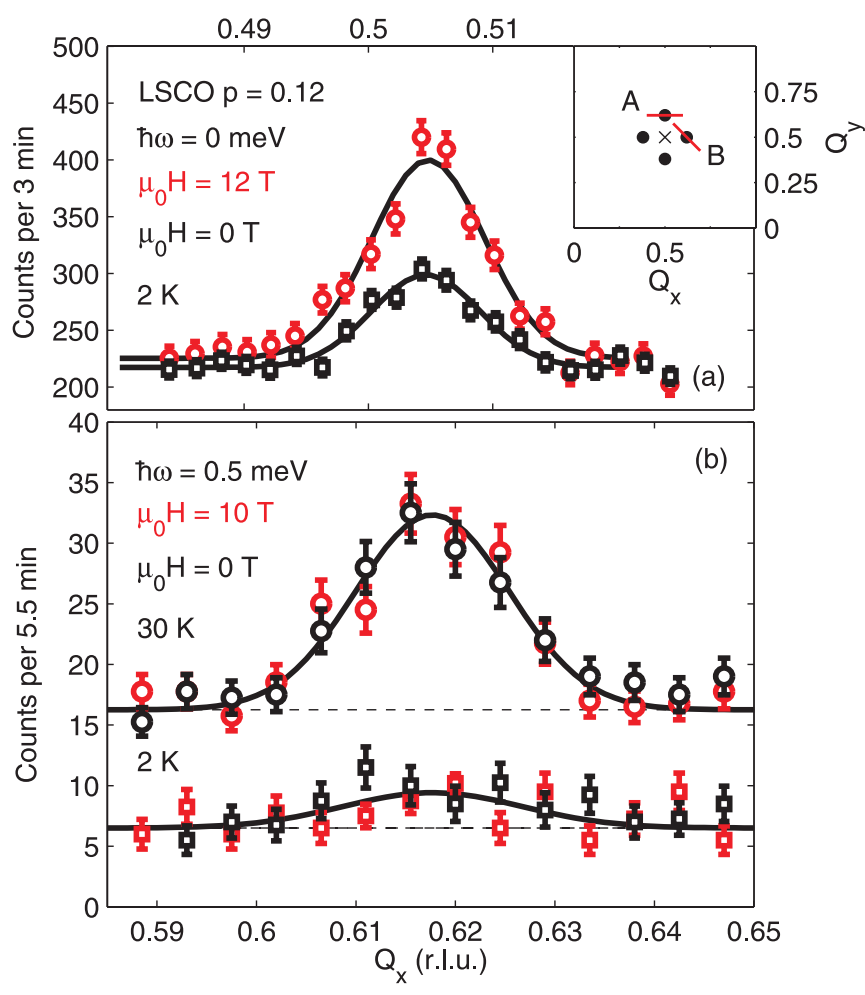

FIG. 1. (Color online) (a) Elastic scattering intensity at an incommensurate position $Q_{\mathrm{IC}}$ as indicated in the inset where circles mark the locii of incommensurate magnetic order and low-energy fluctuations. Black and red data points were taken at $0-\mathrm{T}$ and 12-T field, respectively. Solid lines are Gaussian fits to data. These data are reproduced from Ref. 19. The inset shows the two different scan directions for the elastic scattering data (A) and the inelastic scattering data (B). (b) Background-subtracted intensity for a constant energy scan with $\hbar \omega=0.5 \mathrm{meV}$. Notice that for visibility, the data have an arbitrary offset. Solid lines are Gaussian fits to data. Neither at $2 \mathrm{~K} \ll T_{\mathrm{c}}$ nor in the normal state at $30 \mathrm{~K}>T_{\mathrm{c}}$ does the application of a magnetic field of $10 \mathrm{~T}$ have any effect on the low-energy spin excitations at $\hbar \omega=0.5 \mathrm{meV}$. The nominally elastic and the inelastic peaks are all resolution limited; $\xi_{\text {elastic }} \geqslant 110 \AA$ and $\xi_{0.5 \mathrm{meV}} \geqslant 70 \AA$. 
theorem:

$$
S(\mathbf{Q}, \omega, T)=\chi^{\prime \prime}(\mathbf{Q}, \omega, T) n_{B}(\omega, T) .
$$

where $n_{B}(\omega, T)=\left(1-e^{-\hbar \omega / k_{\mathrm{B}} T}\right)^{-1}$ is the Bose occupation factor. In addition to the magnetic scattering $S(\mathbf{Q}, \omega, T)$, the raw experimental data also contain contributions that do not arise from electronic magnetism, but are due to scattering from atomic nuclei. To obtain $\chi^{\prime \prime}(\mathbf{Q}, \omega, T)$ it is important to cleanly separate these contributions. For the strongly peaked response observed at low energies in LSCO, an effective background subtraction procedure is to estimate the nonmagnetic contributions from the scattering observed at wave vectors sufficiently far away from the magnetic peaks. We studied the magnetic fluctuations over the temperature range $2-80 \mathrm{~K}$ and for energy transfers in the range $0.3-7 \mathrm{meV}$. Most of the results we report were obtained with fixed final neutron energy $E_{\mathrm{f}}=5.0 \mathrm{meV}$. For measurements of spin excitations at energy transfers, $\hbar \omega=0.3-0.5 \mathrm{meV}$, we chose a lower final energy $E_{\mathrm{f}}=4.1 \mathrm{meV}$ to avoid contamination from strictly elastic scattering through the finite energy resolution. In this case, the energy resolution was $0.13 \mathrm{meV}$ FWHM as compared to $0.18 \mathrm{meV}$ at $E_{f}=5.0 \mathrm{meV}$.

\section{RESULTS}

The temperature and magnetic field dependencies of static magnetism in LSCO $p \sim 0.12$, as well as its momentum space characteristics, has previously been studied in great detail. ${ }^{17,19,21,32-34}$ In Fig. 1(a), we show a constant energy scan obtained with the spectrometer set to energy transfer $\hbar \omega=$ $0 \mathrm{meV}$. A momentum-resolution limited peak is observed close to $(1 / 2,1 / 2+\delta, 0)$ when performing a scan in the direction indicated by A in the inset of Fig. 1(a). The slight offset from $Q_{x}=0.5$ r.l.u. is consistent with the observation of Kimura et $a l .{ }^{33}$ that the incommensurate, nominally elastic peaks do not lie along the high-symmetry directions of the underlying $\mathrm{CuO}_{2}$ lattice, but are slightly displaced. In our sample, the magnetic intensity increases significantly when a magnetic field is applied along the crystallographic $c$ axis, see Fig. 1(a) and Ref. 19. The onset temperature for the magnetic order is essentially field independent, as can be seen in Fig. 2(a).

Figure 1(b) displays the inelastic response at $Q_{\text {IC }}$ with energy transfer $\hbar \omega=0.5 \mathrm{meV}$, probed at base temperature and just above $T_{c}$. The scan was performed along the direction indicated by B in the inset of Fig. 1(a). Data taken in zero field and at $H=10 \mathrm{~T}$ are shown. In strong contrast to the elastic response shown in Fig. 1(a), no detectable field effect was observed at any temperature, as seen in Fig. 2(b). The lack of field-effect persists throughout the energy range $0.3-7.0 \mathrm{meV}$ at both $T=2$ and $30 \mathrm{~K}$ as shown in Figs. 2(c) and 2(d), respectively. The peak position was determined by full $Q$ scans as in Fig. 1(b) and was observed to be independent of temperature and energy transfer within the temperature and energy range of this experiment. From the $Q$ scans, we found that the inelastic correlation length is resolution limited by $\xi(T, \omega) \geqslant 70 \AA$ for $\hbar \omega<3 \mathrm{meV}$ and $T<50 \mathrm{~K}$. Further data were therefore taken by three-point scans; counting at the peak position and two background positions on each side of the peak. The background estimates are subtracted from the peak intensities in Figs. 3(a)-3(c) and 4(a). The solid
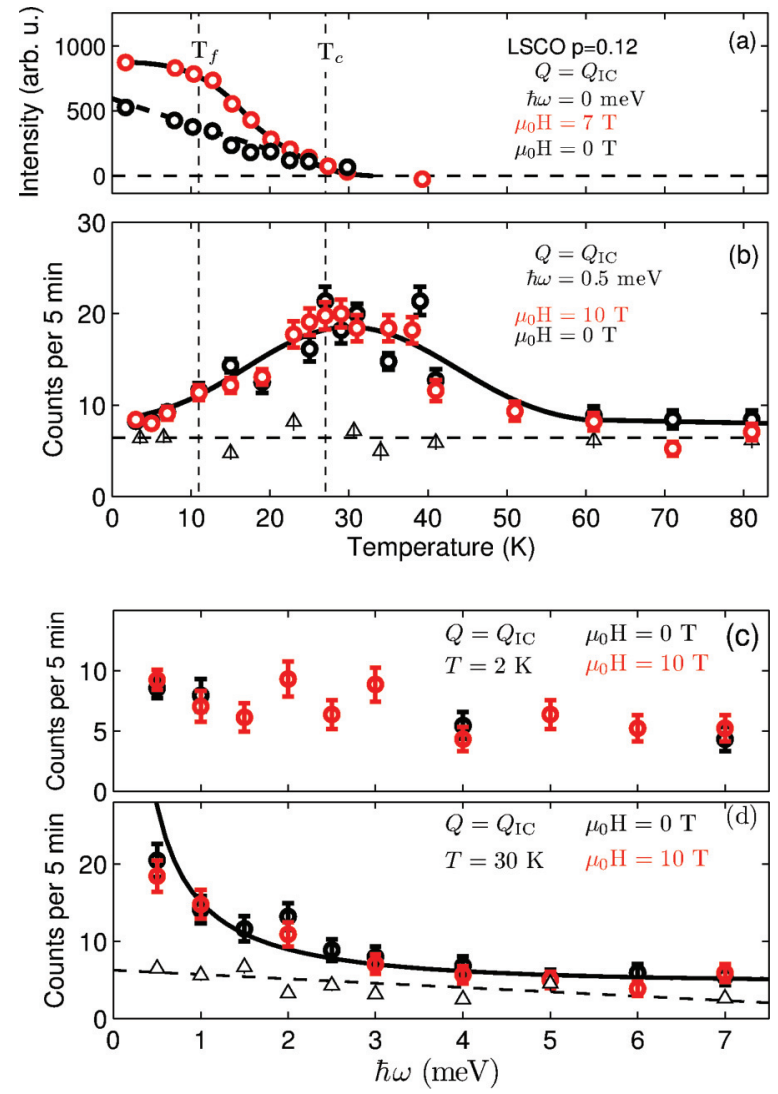

FIG. 2. (Color online) (a) Temperature dependence of the background-subtracted elastic response at $Q_{\mathrm{IC}}$ for $H=0$ (black) and $7 \mathrm{~T}$ (red). The vertical dashed lines indicate the superconducting transition temperature, $T_{\mathrm{c}} \approx 27 \mathrm{~K}$ and the freezing temperature for magnetic ordering, $T_{f} \approx 11 \mathrm{~K}$, obtained from muon spin rotation. ${ }^{30}$ (b) Temperature dependence of the inelastic response $(0.5 \mathrm{meV})$ at $Q_{\text {IC }}$ in zero field (black) and $10 \mathrm{~T}$ (red). Open triangles are background data. (c) and (d) Inelastic response at $Q_{\mathrm{IC}}$ as a function of energy in the superconducting state at $T=2 \mathrm{~K}$ and normal phase at $T=30 \mathrm{~K}$ both with (red points) and without (black points) an applied field of $10 \mathrm{~T}$. Open triangles are background data. The dashed line is a fit to a linear function. The solid black line in (d) is a fit to the Bose occupation factor as described in the text.

line in Fig. 2(d) is the Bose occupation factor $n_{B}(\omega, T)$ scaled to the data. This lead us to the conclusion that the energy dependence of $S\left(\mathbf{Q}_{\mathrm{IC}}, \omega, T\right)$ at $30 \mathrm{~K}$ is dominantly given by the Bose occupation factor for energies in the range 1-7 meV, and hence that $\chi^{\prime \prime}\left(\mathbf{Q}_{\mathrm{IC}}, \omega, T\right)$ is roughly frequency independent.

To elucidate the details of the temperature dependence, we show in Fig. 3 the temperature dependence of the inelastic response for three different energies from 0.3 to $1.5 \mathrm{meV}$. As in Fig. 2(b), we observe a broad maximum around $T_{\mathrm{c}}$. The position of the maximum shifts down in temperature as $\hbar \omega$ is decreased, and approaches $T_{c}$ in the limit $\hbar \omega \rightarrow 0 \mathrm{meV}$. For all energy transfers probed, the intensity decreases as the sample is cooled from $T_{c}$ to base temperature. This tendency is much less pronounced for $1.5 \mathrm{meV}$ than for $0.3 \mathrm{meV}$. At the former, the intensity remains finite in the low-temperature limit, whereas it approaches zero for the latter.

To investigate how the suppression of intensity at very low energies $(\hbar \omega<1 \mathrm{meV})$ manifests itself in the observed 


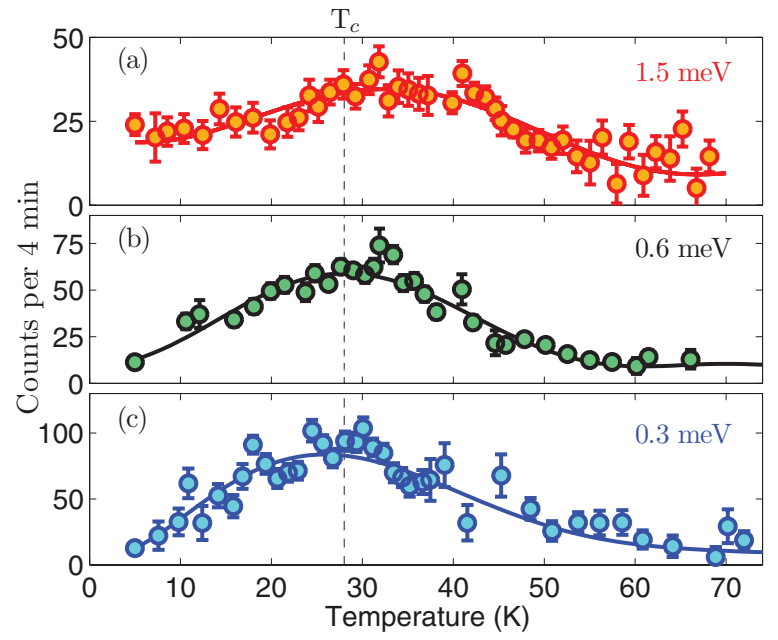

FIG. 3. (Color online) Background-subtracted inelastic response at $Q=Q_{\text {IC }}$ in zero field and plotted as intensity versus temperature for excitation energies: (a) 1.5 , (b) 0.6 , and (c) $0.3 \mathrm{meV}$. Vertical dashed line indicates the $T_{\mathrm{c}}$ of this compound and solid lines are guides to the eye. Since these measurements were obtained without a magnet there is a significant increase in intensity compared to the data shown in Figs. 1 and 2.

spectra, we show in Fig. 4 the energy dependence of the incommensurate signal for several temperatures above and below $T_{\mathrm{c}}$. Figure 4(a) shows how the intensity is drastically reduced for energy tranfers lower than $0.75 \mathrm{meV}$ at $T=5$ $\mathrm{K}$ and, to a lesser extent, for $T=13 \mathrm{~K}$. This shows that $\chi^{\prime \prime}\left(\mathbf{Q}_{\mathrm{IC}}, \omega, T\right)$ becomes frequency-dependent for temperatures lower than and comparable to the freezing temperature $T_{f} \approx$ $11 \mathrm{~K}$ deduced from $\mu \mathrm{SR}$. The guide to the eye for the $5 \mathrm{~K}$ data suggests an interpretation in terms of two energy gaps. We return to this point in the discussion.

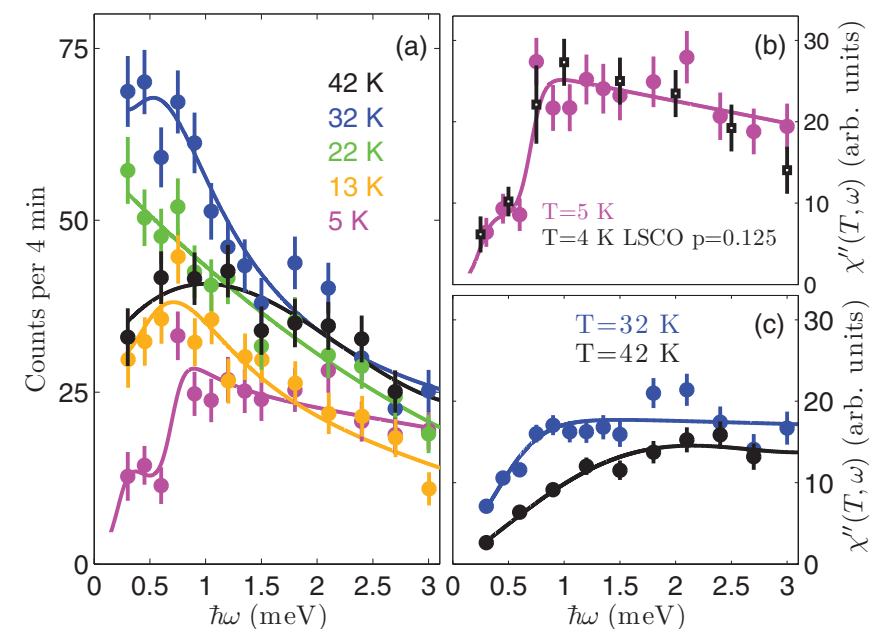

FIG. 4. (Color online) (a) Background-subtracted inelastic response as a function of excitation energy at different temperatures $T$. (b) Dynamical susceptibility $\chi^{\prime \prime}\left(\mathbf{Q}_{\mathrm{IC}}, \omega, T\right)$ at $T=5 \mathrm{~K}$ obtained for our $p=0.12$ sample and compared to low-temperature data reproduced from M. Kofu et al. ${ }^{21}$ for their $p=0.125$ sample at $T=4 \mathrm{~K}$ (c) Dynamical susceptibility $\chi^{\prime \prime}\left(\mathbf{Q}_{\mathrm{IC}}, \omega, T\right)$ for fixed $T=32$ and $42 \mathrm{~K}$. All lines are guides to the eye.
To illustrate the effects of the Bose occupation factor, see Eq. (1), we plot the corresponding dynamic susceptibilities in Figs. 4(b) and 4(c) for three temperatures: 5, 32, and $42 \mathrm{~K}$. By contrast to the smooth energy dependence of the susceptibility at high temperatures, the 5-K data display an abrupt reduction by a factor of roughly four below $\hbar \omega \sim 0.75 \mathrm{meV}$, see Fig. 4(b). This observation is consistent with measurements at a similar doping level, $p=0.125$, by M. Kofu et $a .^{21}$ We stress that the intensity suppression is only partial even at the lowest frequency, as seen in Figs. 3(c) and 4(b), but signatures of gapping of the spectrum are clear from Figs. 4, 3(b), 3(c), and 2(b). Moreover, from Fig. 2(b), it is apparent that these gap signatures do not exhibit any magnetic field dependence.

\section{DISCUSSION}

In this section, we first discuss the temperature dependence of the inelastic data and indications of glassy dynamics. Thereafter we turn to the energy dependence and the intensity suppression observed at low energy transfers. Finally, we discuss the field dependence of the nominally elastic data and the field independence of the inelastic data.

\section{A. Freezing and glassiness}

The peaked response of the low-energy fluctuations shown in Figs. 2(b) and 3 is a common feature observed throughout the doping range in LSCO. ${ }^{23,26,35}$ We observe a peak in $S\left(\mathbf{Q}_{\mathrm{IC}}, \omega, T\right)$ at temperatures close to $T_{c}$. The peak shifts towards higher temperatures as the energy transfer, $\hbar \omega$, increases. This shift, consistent with glassy behavior, was also observed in very underdoped, nonsuperconducting $\operatorname{LSCO} p=$ 0.04 (see Ref. 36). In that case, the integrated spin intensity peaked at a temperature that increased for increasing energy; the peak temperature was given by $T \sim 2 \hbar \omega$. In our case, we probe only low-energy transfers, but the tendency is the same, i.e., the threshold temperature above which the intensity drops off increases with frequency, see Fig. 3. We stress that since the observed peak widths are roughly constant for the range of temperatures and energy transfers probed in our experiments, the peak amplitude at $Q_{\text {IC }}$ is to a good approximation proportional to the integrated intensity discussed in Ref. 36. We therefore conclude that our $\mathrm{La}_{1.88} \mathrm{Sr}_{0.12} \mathrm{CuO}_{4}$ crystal displays low-energy dynamics that are similar to what was found in the glassy ground state of very underdoped LSCO $p=0.04 .^{36-38}$

Turning to magnetic order, we observe a nominally elastic signal for $T<T_{c}$, see Fig. 2. For an energy resolution of $\Delta E \sim 0.18 \mathrm{meV}$, the time resolution $t \sim \frac{\hbar}{\Delta E}$ is of the order tens of picoseconds. Fluctuations with a characteristic time scale slower than picoseconds will therefore appear as static. For our crystal, the freezing temperature obtained by zero field $\mu \mathrm{SR}$, for which the time resolution is of the order of microseconds, is $T_{f} \approx 11 \mathrm{~K} .^{30}$ This implies that the magnetic ordering temperature as observed by neutron scattering is comparable to $T_{c}$ only by a coincidence. Further, it implies that the nominally elastic neutron scattering signal observed in the temperature range between $T_{f}$ and $T_{c}$ is actually caused by strictly inelastic, low-energy magnetic fluctuations picked up by the experimental resolution function of the spectrometer, i.e., fluctuations with characteristic energy scale $0.18 \mathrm{meV}$ or lower. Note also that $T_{f}$ obtained by $\mu \mathrm{SR}$ only sets an upper 
limit of the actual freezing temperature: an experimental technique probing the spin dynamics on a longer characteristic timescale than microseconds could give an even lower value.

\section{B. Anisotropy gap}

Figure 4 shows that for temperatures lower than $T_{f}$, we observe a partial suppression of low-energy fluctuations. There is an intensity drop at energy transfers lower than $\hbar \omega \sim$ $0.75 \mathrm{meV}$. Below this scale, we do not see a spectral region of zero intensity, which means that the gap is not fully developed. We now turn to discuss the possible origin of these observations within a simple spin wave formalism. The energy gaps in the parent compound $\mathrm{La}_{2} \mathrm{CuO}_{4}$ are due to exchange anisotropy and the Dzyaloshinskii-Moriya interaction. These gaps were reported by C. J. Peters et al. ${ }^{39}$ to be 1.0 and $2.5 \mathrm{meV}$ for the in-plane and out-of-plane gaps, respectively. Within the standard Heisenberg spin-only approach, an anisotropy gap is expected to scale with the ordered magnetic moment of the $\mathrm{Cu}$ atoms. This is a generic result also expected to hold for stripeordered systems for which the static moments are known to be strongly diminished compared to $\mathrm{La}_{2} \mathrm{CuO}_{4} \cdot{ }^{40}$ The ordered moment in our sample was previously determined to be an order of magnitude smaller than that of $\mathrm{La}_{2} \mathrm{CuO}_{4} \cdot{ }^{19}$ Therefore, ignoring any additional effects due to quenched disorder produced by the replacement of $\mathrm{La}$ by $\mathrm{Sr}$, we can expect anisotropy gaps in $\mathrm{La}_{1.88} \mathrm{Sr}_{0.12} \mathrm{CuO}_{4}$ to be roughly an order of magnitude smaller than in $\mathrm{La}_{2} \mathrm{CuO}_{4}$. This would make the gaps comparable to the energy resolution of our experiments. An interpretation of our data, which is consistent with these qualitative arguments, is that we observe the out-of-plane energy gap at $\hbar \omega \sim 0.75 \mathrm{meV}$, while our experiment does not resolve the smaller in-plane gap. This explains why we observe only a partial suppression of the scattering signal rather than a fully developed energy gap. Note, however, that experiments probing spin fluctuations at a single incommensurate wave vector only do not allow us to directly identify the larger gap as due to out-of-plane anisotropy rather than in-plane anisotropy. Evidence for a residual small spin anisotropy gap was also discussed for a $p=0.04$ sample in the nonsuperconducting spin-glass regime of LSCO. ${ }^{36}$

Turning to stripe-ordered $\mathrm{La}_{1.875} \mathrm{Ba}_{0.125} \mathrm{CuO}_{4}$, a low-energy intensity suppression of the same magnitude below $0.7 \mathrm{meV}$ was recently observed ${ }^{41,42}$ and similarly ascribed to spin anisotropy of the spin-ordered state. In that case, too, a magnetic field effect of the low-energy excitations was absent. Hence, we find striking similarities for the low-energy excitations between our sample and $\mathrm{La}_{1.875} \mathrm{Ba}_{0.125} \mathrm{CuO}_{4}$, indicating that both samples have similar magnetic regions and anisotropy gaps.

The nickelates, $\mathrm{La}_{2-x} \mathrm{Sr}_{x} \mathrm{NiO}_{4}$, also show a stripe-ordered phase upon doping with regions of antiferromagnetically ordered spins separated by lines of holes. Although nickelates do not become superconducting upon doping and also do not display the hourglass dispersion common to La-based cuprates ${ }^{27,43}$ and $\mathrm{La}_{2-x} \mathrm{Sr}_{x} \mathrm{CoO}_{4},{ }^{44}$ the existence of stripe order in both justify a qualitative comparison. Studies of the low-energy magnetic dynamics in nickelates over a broad range of $\mathrm{Sr}$ content have shown evidence of an out-of-plane anisotropy gap which decreases with increasing doping. ${ }^{45,46}$
The decrease is about a factor of two comparing the parent compound $\mathrm{La}_{2} \mathrm{NiO}_{4}{ }^{47}$ to doping $x=0.275-0.37$ (see Ref. 46) and roughly a factor of three for doping values $x=0.4-0.45 .^{48}$ This trend is similar to the behavior we have identified in $\mathrm{La}_{1.88} \mathrm{Sr}_{0.12} \mathrm{CuO}_{4}$. Summarizing the above, it appears that the low-energy spin dynamics in underdoped La-based cuprates and nickelates can display small residual anisotropy gaps, irrespective of whether they are stripe-ordered as $\mathrm{La}_{1.875} \mathrm{Ba}_{0.125} \mathrm{CuO}_{4}{ }^{41,42}$ and $\mathrm{La}_{2-x} \mathrm{Sr}_{x} \mathrm{NiO}_{4}$ or undergoing glassy freezing as in $\mathrm{La}_{2-x} \mathrm{Sr}_{x} \mathrm{CuO}_{4}$.

M. Kofu et al. ${ }^{21}$ recently reported a study of the low-energy excitations in LSCO at doping values $p=0.125-0.14$. The results were discussed in terms of a two-component scenario with a real space separation of two phases for $p \leqslant 0.13$. In the latter regime, spin fluctuations of short correlation length were proposed to exist at energies above an energy scale $E_{g}$ comparable to the spin gap observed at optimal doping, and to coexist with low-energy fluctuations that have significantly longer correlations length. Our data do not allow us to confirm or dismiss a change in correlation length with energy transfer. We note, on the other hand, that a partial suppression of low-energy spectral weight below an energy scale $\sim 1.0 \mathrm{meV}$ was detected by M. Kofu et al. in their $p=0.125$ sample [see Fig 4(b)]. Moreover, no suppression was seen for $p=$ $0.13,{ }^{21}$ which resides at the edge of magnetic order ${ }^{20}$ and should therefore have a much smaller ordered moment and hence much smaller anisotropy gaps. Both observations are consistent with our interpretation of the low-energy intensity suppression as originating from an anisotropy gap.

\section{Magnetic field effect}

An intriguing difference becomes apparent when comparing the magnetic field effect of the nominally elastic [see Figs. 1(a) and 2(a)] and inelastic [see Figs. 1(b) and 2(b)-2(d)] signals. Due to the finite energy resolution of a neutron experiment, we conclude from a comparison of Fig. 2(a) with $\mu \mathrm{SR}$ results that there is a significant field effect in the very low-energy fluctuations ( $\hbar \omega<0.18 \mathrm{meV}$ ) for temperatures below $T \sim 25 \mathrm{~K}$. This effect stands in contrast to the fieldindependent magnetic response in the energy range $0.5-7 \mathrm{meV}$. The absence of a field effect in this range is distinctly different from the behavior observed for compositions with slightly smaller $^{23}$ as well as higher hole doping, ${ }^{24,26-28}$ which exhibit a magnetic-field enhancement of the spectral weight in this energy range for temperatures below $T_{\mathrm{c}}$.

In a spin-only Heisenberg approach, where we account for an easy axis and Dzyaloshinskii-Moriya anisotropies, we can estimate the effect of an applied magnetic field $H$ on the anisotropy gap at the antiferromagnetic ordering vector. Due to the Dzyaloshinskii-Moriya interaction the spins tilt slightly out of the $\mathrm{CuO}_{2}$ planes. In the mathematical expression for the energy gap, the applied magnetic field $H$ enters in a term that multiplies the small tilting angle. Therefore the energy gaps are only weakly dependent on $H$, and we estimate an energy change due to an applied field of $\mu_{0} H=10 \mathrm{~T}$ of the same order of magnitude as our energy resolution. In conclusion, at the lowest temperatures, we do not necessarily expect to detect any significant effect of a magnetic field on the anisotropy gaps. This is consistent with our observations in $\mathrm{La}_{1.88} \mathrm{Sr}_{0.12} \mathrm{CuO}_{4}$. 
Returning to the lowest energy ( $\hbar \omega<0.18 \mathrm{meV}$ ) spin excitations in $\mathrm{La}_{1.88} \mathrm{Sr}_{0.12} \mathrm{CuO}_{4}$ and their enhancement in a magnetic field, further experiments with improved energy resolution will be required to determine if they are of an origin distinct from the spin-wave-like excitations observed at higher energies, or whether - as the above arguments suggest - they are related to the anisotropy gap not resolved by the present experiment.

The observation of a magnetic field-effect for $p=0.105$ and its interpretation as a renormalized superconducting spin-gap $^{23}$ may now be rationalized by the existence of a sharp peak around $x_{\max }=0.12$ in the freezing temperature $T_{f} .{ }^{20}$ The peak in the freezing temperature is associated with an increased competition between static magnetic order and superconductivity and a decrease of $T_{c} .{ }^{16}$ We have argued that for $p=0.12$, the spin-frozen low-temperature state permits an anisotropy gap akin to that observed in the parent insulator $\mathrm{La}_{2} \mathrm{CuO}_{4}$. Conversely, when the magnetic ordering/freezing tendencies are weakened by moving to away from $x_{\max }$, the low-energy dynamics can be expected to become dominated by the physics of the superconductor with its superconducting spin gap opening at $T_{c}$, rather than that of the insulator with its anisotropy gaps. This physical picture goes a long way towards reconciling the disagreements of interpretations between J. Chang et al. ${ }^{23}$ and M. Kofu et al. ${ }^{21}$

\section{CONCLUSIONS}

We have studied the detailed temperature and energy dependence of low-energy magnetic fluctuations in $\mathrm{La}_{1.88} \mathrm{Sr}_{0.12} \mathrm{CuO}_{4}$. A discrepancy between the magnetic ordering temperature derived by neutrons and muons shows that the spins undergo freezing rather than a regular phase transition. We find additional evidence for freezing in the temperature dependence of the low-energy fluctuations, which resembles the behavior observed in LSCO at much lower doping, in the non-superconducting, so-called spin-glass regime, and therefore conclude that even superconducting $\mathrm{La}_{1.88} \mathrm{Sr}_{0.12} \mathrm{CuO}_{4}$ exhibits spin glass behavior.

Below the spin-freezing temperature $T_{f} \approx 11 \mathrm{~K}$ of our sample, we have detected an incomplete suppression of magnetic spectral weight at energies larger than our energy resolution, $\Delta E$, but smaller than $0.75 \mathrm{meV}$. We ascribe this effect to the development of a spin anisotropy gap in a spin frozen setting. Applying insights from spin wave theory, this interpretation is supported by the insensitivity, to within our experimental resolution, of the low-energy intensity suppression to an applied magnetic field of $10 \mathrm{~T}$. It is notable that the low-energy excitations in our $\mathrm{La}_{1.88} \mathrm{Sr}_{0.12} \mathrm{CuO}_{4}$ sample are remarkably similar to those of $\mathrm{La}_{1.875} \mathrm{Ba}_{0.125} \mathrm{CuO}_{4}$, which displays long-range spin and charge stripe order.

In contrast to the insensitivity of the anisotropy gap to applied magnetic field, a significant enhancement of nominally elastic, incommensurate magnetic signal was observed at temperatures lower than $T_{\mathrm{c}}$. Given that muon spin rotation yields a freezing temperature $T_{f}$ much smaller than $T_{c}$, the implication is that there must be a magnetic field effect on remnant spin excitations at energies inside our resolution window $\Delta E \simeq 0.18 \mathrm{meV}$.

Our experimental data and a comparison with published data on LBCO and LSCO have illuminated that the sharp maximum in the spin freezing temperature $T_{f}$ near $p=0.12$ is reflected equally dramatically in the lowest-energy magnetic excitations, as the superconducting gap observed at optimal doping is replaced by a spin anisotropy gap sufficiently close to the maximum in $T_{f}$.

\section{ACKNOWLEDGMENTS}

We acknowledge Paul G. Freeman for illuminating discussions on similarities between cuprates and nickelates. We thank RITA-II, SINQ at the Paul Scherrer Institute, Switzerland, for the technical support of our experiments. This work was supported by the Swiss NSF (through NCCR, MaNEP, and Grant Nos. 200020-105151, 200020-130522, PBEZP2122855, PZ00P2-142434), by the Ministry of Education and Science of Japan, and by the Danish Council for Independent Research in Natural Sciences (FNU) through DANSCATT and the grant Magnetism in Superconductors. The present experiments were performed at the FRM-II research reactor and were supported by the European Commission under the 7th Framework program through the "Research Infrastructures" action of the "Capacities" program, Contract No. CP-CSA INFRA-2008-1.1.1 Number 226507-NMI3.
${ }^{1}$ E. Dagotto, Science 309, 257 (2005).

${ }^{2}$ J. G. Bednorz and K. A. Müller, Z. Phys. B 64, 188 (1986).

${ }^{3}$ B. Fauqué, Y. Sidis, V. Hinkov, S. Pailhès, C. T. Lin, X. Chaud, and P. Bourges, Phys. Rev. Lett. 96, 197001 (2006).

${ }^{4}$ V. Balédent, B. Fauqué, Y. Sidis, N. B. Christensen, S. Pailhès, K. Conder, E. Pomjakushina, J. Mesot, and P. Bourges, Phys. Rev. Lett. 105, 027004 (2010).

${ }^{5}$ S. Li, Z. Yamani, H. J. Kang, K. Segawa, Y. Ando, X. Yao, H. A. Mook, and P. Dai, Phys. Rev. B 77, 014523 (2008).

${ }^{6}$ M. Fujita, H. Goka, K. Yamada, J. M. Tranquada, and L. P. Regnault, Phys. Rev. B 70, 104517 (2004).

${ }^{7}$ M. Hücker, M. v. Zimmermann, G. D. Gu, Z. J. Zu, J. S. Wen, G. Xu, H. J. Kang, A. Zheludev, and J. M. Tranquada, Phys. Rev. B 83, 104506 (2011).
${ }^{8}$ J. M. Tranquada, B. J. Sternlieb, J. D. Axe, Y. Nakamura, and

S. Uchida, Nature (London) 375, 561 (1995).

${ }^{9}$ G. Ghiringhelli et al., Science 337, 821 (2012).

${ }^{10}$ J. Chang, E. Blackburn, A. T. Holmes, N. B. Christensen, J. Larsen, J. Mesot, Ruixing Liang, D. A. Bonn, W. N. Hardy, A. Watenphul, M. V. Zimmermann, E. M. Forgan, S. M. Hayden, Nat. Phys. 8, 871 (2012).

${ }^{11}$ H.-H. Klauss, W. Wagener, M. Hillberg, W. Kopmann, H. Walf, F. J. Litterst, M. Hücker, and B. Büchner, Phys. Rev. Lett. 85, 4590 (2000).

${ }^{12}$ J. Fink, E. Schierle, E. Weschke, J. Geck, D. Hawthorn, V. Soltwisch, H. Wadati, H.-H. Wu, H. A. Dürr, N. Wizent, B. Büchner, and G. A. Sawatzky, Phys. Rev. B 79, 100502(R) (2009). 
${ }^{13}$ R. Liang, D. A. Bonn, and W. N. Hardy, Phys. Rev. B 73, 180505(R) (2006).

${ }^{14}$ T. Wu, H. Mayaffre, S. Krämer, M. Horvatić, C. Berthier, W. N. Hardy, R. Liang, D. A. Bonn, and M.-H. Julien, Nature (London) 477, 191 (2011).

${ }^{15}$ F. LaLiberté, J. Chang, N. Doiron-Leyraud, E. Hassinger, R. Daou, M. Rondeau, B. J. Ramshaw, R. Liang, D. A. Bonn, W. N. Hardy, S. Pyon, T. Takayama, H. Takagi, I. Sheikin, L. Malone, C. Proust, K. Behnia, and L. Taillefer, Nat. Commun. 2, 432 (2011).

${ }^{16}$ H. Takagi, T. Ido, S. Ishibashi, M. Uota, S. Uchida, and Y. Tokura, Phys. Rev. B 40, 2254 (1989).

${ }^{17}$ T. Suzuki, T. Goto, K. Chiba, T. Shinoda, T. Fukase, H. Kimura, K. Yamada, M. Ohashi, and Y. Yamaguchi, Phys. Rev. B 57, R3229 (1998).

${ }^{18}$ H.-H. Wu, M. Buchholz, C. Trabant, C. F. Chang, A. C. Komarek, F. Heigl, M. v. Zimmermann, M. Cwik, F. Nakamura, M. Braden, and C. Shüßler-Langeheine, Nat. Commun. 3, 1023 (2012).

${ }^{19}$ J. Chang, Ch. Niedermayer, R. Gilardi, N. B. Christensen, H. M. Rønnow, D. F. McMorrow, M. Ay, J. Stahn, O. Sobolev, A. Hiess, S. Pailhes, C. Baines, N. Momono, M. Oda, M. Ido, and J. Mesot, Phys. Rev. B 78, 104525 (2008).

${ }^{20}$ M.-H. Julien, Physica B 329-333, 693 (2003).

${ }^{21}$ M. Kofu, S.-H. Lee, M. Fujita, H.-J. Kang, H. Eisaki, and K. Yamada, Phys. Rev. Lett. 102, 047001 (2009).

${ }^{22}$ T. E. Mason, G. Aeppli, and H. A. Mook, Phys. Rev. Lett. 68, 1414 (1992).

${ }^{23}$ J. Chang, A. P. Schnyder, R. Gilardi, H. M. Rønnow, S. Pailhes, N. B. Christensen, Ch. Niedermayer, D. F. McMorrow, A. Hiess, A. Stunault, M. Enderle, B. Lake, O. Sobolev, N. Momono, M. Oda, M. Ido, C. Mudry, and J. Mesot, Phys. Rev. Lett. 98, 077004 (2007).

${ }^{24}$ J. Chang, N. B. Christensen, Ch. Niedermayer, K. Lefmann, H. M. Rønnow, D. F. McMorrow, A. Schneidewind, P. Link, A. Hiess, M. Boehm, R. Mottl, S. Pailhés, N. Momono, M. Oda, M. Ido, and J. Mesot, Phys. Rev. Lett. 102, 177006 (2009).

${ }^{25}$ N. B. Christensen, J. Chang, E. Razzoli, M. Bator, Ch. Niedermayer, K. Lefmann, H. M. Ronnow, D. F. Mcmorrow, A. Schneidewind, P. Link, A. Hiess, M. Boehm, R. Mottl, S. Pailhès, M. Oda, M. Ido, N. Momono, and J. Mesot, J. Phys. Soc. Jpn. Suppl. B 80, SB030 (2011),

${ }^{26}$ B. Lake, G. Aeppli, K. N. Clausen, D. F. McMorrow, K. Lefmann, N. E. Hussey, N. Mangkorntong, M. Nohara, H. Takagi, T. E. Mason, and A. Schröder, Science 291, 1759 (2001).

${ }^{27}$ J. M. Tranquada, C. H. Lee, K. Yamada, Y. S. Lee, L. P. Regnault, and H. M. Ronnow, Phys. Rev. B 69, 174507 (2004).

${ }^{28}$ R. Gilardi, A. Hiess, N. Momono, M. Oda, M. Ido, and J. Mesot, Europhys. Lett. 66, 840 (2004).

${ }^{29}$ T. Nakano, N. Momono, M. Oda, and M. Ido, J. Phys. Soc. Jpn. 67, 2622 (1998).
${ }^{30} \mathrm{~J}$. Larsen et al. (to be published).

${ }^{31}$ D. Vaknin, S. K. Sinha, D. E. Moncton, D. C. Johnston, J. M. Newsam, C. R. Safinya, and H. E. King, Jr., Phys. Rev. Lett. 58, 2802 (1987).

${ }^{32}$ B. Lake, H. M. Rønnow, N. B. Christensen, G. Aeppli, K. Lefmann, D. F. McMorrow, P. Vorderwisch, P. Smeibidl, N. Mangkorntong, T. Sasagawa, M. Nohara, H. Takagi, and T. E. Mason, Nature 415, 299 (2002).

${ }^{33}$ H. Kimura, H. Matsushita, K. Hirota, Y. Endoh, K. Yamada, G. Shirane, Y. S. Lee, M. A. Kastner, and R. J. Birgeneau, Phys. Rev. B 61, 14366 (2000).

${ }^{34}$ S. Katano, M. Sato, K. Yamada, T. Suzuki, and T. Fukase, Phys. Rev. B 62, R14677 (2000).

${ }^{35}$ K. Yamada, S. Wakimoto, G. Shirane, C. H. Lee, M. A. Kastner, S. Hosoya, M. Greven, Y. Endoh, and R. J. Birgeneau, Phys. Rev. Lett. 75, 1626 (1995).

${ }^{36}$ B. Keimer, N. Belk, R. J. Birgeneau, A. Cassanho, C. Y. Chen, M. Greven, M. A. Kastner, A. Aharony, Y. Endoh, R. W. Erwin, and G. Shirane, Phys. Rev. B 46, 14034 (1992).

${ }^{37}$ F. C. Chou, N. R. Belk, M. A. Kastner, R. J. Birgeneau, and A. Aharony, Phys. Rev. Lett. 75, 2204 (1995).

${ }^{38}$ S. Wakimoto, S. Ueki, Y. Endoh, and K. Yamada, Phys. Rev. B 62, 3547 (2000).

${ }^{39}$ C. J. Peters, R. J. Birgeneau, M. A. Kastner, H. Yoshizawa, Y. Endoh, J. Tranquada, G. Shirane, Y. Hidaka, M. Oda, M. Suzuki, and T. Murakami, Phys. Rev. B 37, 9761 (1988).

${ }^{40}$ S. Wakimoto, R. J. Birgeneau, Y. S. Lee, and G. Shirane, Phys. Rev. B 63, 172501 (2001).

${ }^{41}$ J. M. Tranquada, G. D. Gu, M. Hücker, Q. Jie, H.-J. Kang, R. Klingeler, Q. Li, N. Tristan, J. S. Wen, G. Y. Xu, Z. J. $\mathrm{Xu}$, J. Zhou, and M. v. Zimmermann, Phys. Rev. B 78, 174529 (2008).

${ }^{42}$ J. Wen, Z. Xu, G. Xu, J. M. Tranquada, G. Gu, S. Chang, and H. J. Kang, Phys. Rev. B 78, 212506 (2008).

${ }^{43}$ N. B. Christensen, D. F. McMorrow, H. M. Rønnow, B. Lake, S. M. Hayden, G. Aeppli, T. G. Perring, M. Mangkorntong, M. Nohara, and H. Takagi, Phys. Rev. Lett. 93, 147002 (2004).

${ }^{44}$ A. T. Boothroyd, P. Babkevich, D. Prabhakaran, and P. G. Freeman, Nature (London) 471, 341 (2011).

${ }^{45}$ A. T. Boothroyd, D. Prabhakaran, P. G. Freeman, S. J. S. Lister, M. Enderle, A. Hiess, and J. Kulda, Phys. Rev. B 67, 100407(R) (2003).

${ }^{46}$ A. T. Boothroyd, P. G. Freeman, D. Prabhakaran, M. Enderle, and J. Kulda, Physica B 345, 1 (2004).

${ }^{47}$ K. Nakajima, K. Yamada, S. Hosoya, T. Omata, and Y. Endoh, J. Phys. Soc. Jpn. 62, 4438 (1993).

${ }^{48}$ Paul G. Freeman (private communication). 